\title{
DHZ-Fortbildungspunkte: Unterstützung durch noch mehr Fachverbände
}

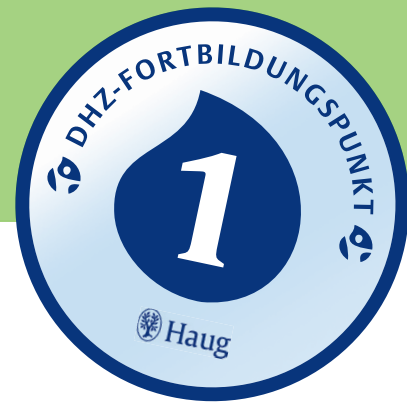

Viele Leser der DHZ sammeln DHZFortbildungspunkte für das freiwillige Fortbildungszertifikat für Heilpraktiker. Seit 2010 unterstützen folgende Fachverbände die FobiPunkte der DHZ als Nachweis für die kontinuierliche Fortbildung an: BDH, AGAHP, BDC, BKHD, HP-Gesellschaft für Elektroakupunktur nach Voll e.V., $\mathrm{HPGO}_{3}$, SHZ und VKHD.

Heilpraktiker bilden sich sehr engagiert und regelmäßig fort - ganz ohne Zwang und Pflicht, einfach, weil sie wissen, dass sie dies ihren Patienten schuldig sind und eine Praxis sich heutzutage auch kaum anders erfolgreich führen ließe. Doch tatsächlich sind sie nach der Berufsordnung für Heilpraktiker (BOH, Artikel 5) zur ständigen Fortbildung verpflichtet. Diese Fortbildung müssen sie zudem nachweisen - im Extremfall vor Gericht.

Ganz unabhängig davon ist eine kontinuierliche Qualifizierung heutzutage unerlässlich. Nicht nur Politiker und maßgebliche Vertreter des Gesundheitswesens fordern eine dokumentierte Qualitätssicherung. Moderne, aufgeklärte Patienten erwarten ebenfalls - völlig zu Recht - aktualisiertes Wissen und umfassende Kompetenz auf neuestem Stand von ihren Therapeuten.

\section{Fortbildungszertifikate - längst} Alltag in vielen Medizinberufen

Viele medizinische Berufe haben inzwischen ein eingeführtes Fortbildungsprogramm, das kontinuierliches Weiterlernen fordert, dokumentiert, zertifiziert und nicht zuletzt auch das Ansehen des Zerti- fizierten steigert. Das CME-System (continuing medical education) der Ärzte hat sich weltweit durchgesetzt. Aber auch die Apotheker, pharmazeutisch-technische Assistenten, Pflegekräfte, Physiotherapeuten und einige Heilpraktikerverbände haben die Notwendigkeit der kontinuierlichen Fortbildung erkannt und inzwischen ihre eigenen Fortbildungszertifikate entwickelt. Gerade vor dem Hintergrund der Berufsstandssicherung ist der Nachweis von qualifizierter Fortbildung von zentraler Bedeutung. So bietet z. B. der Bund Deutscher Heilpraktiker (BDH) gemeinsam mit AGAHP, BDC, BKHD, HP-Gesellschaft für Elektroakupunktur nach Voll e.V., $\mathrm{HPGO}_{3}$, SHZ und VKHD ein freiwilliges Fortbildungszertifikat für Heilpraktiker an. Diese Institutionen nutzen die DHZ-Fortbildungspunkte, um ihren Mitgliedern interaktive Fortbildung zu ermöglichen.

\section{DHZ-Punkte sammeln für das Zertifikat}

Das freiwillige Fortbildungszertifikat für Heilpraktiker der genannten Fachverbände basiert im Wesentlichen auf dem oben beschriebenen generell anerkannten Fortbildungsmodell (CME) und garantiert so die Akzeptanz durch Öffentlichkeit, andere Gesundheitsberufe und die Politik. Die Grundeinheit dieser Fortbildungsaktivitäten ist der Fortbildungspunkt (FP). Dieser entspricht in der Regel einer abgeschlossenen Fortbildungsstunde (45 min). Für die erworbenen Fortbildungspunkte muss ein Nachweis erbracht werden. Punkte können z. B. entweder durch den Besuch von Fortbildungsveranstaltungen, Kongressen, Fachtagungen oder Work- shops gesammelt oder über sog. interaktive Fachfortbildungen durch Internet oder Fachzeitschriften erworben werden. Bei der interaktiven Fachfortbildung sind der Nachweis der Qualifizierung und die schriftliche Auswertung des Lernerfolgs eine Grundvoraussetzung für die Vergabe der Fortbildungspunkte.

Das Fortbildungszertifikat für Heilpraktiker wird ausgestellt, wenn Sie in 3 Jahren 120 Fortbildungspunkte erworben, diese dokumentiert und einen entsprechenden Antrag auf Zertifizierung bei der Dokumentationsstelle des BDH gestellt haben. Davon können Sie maximal 10 Punkte pro Jahr für das Zertifikat mithilfe der interaktiven Fortbildung durch Fachzeitschriften sammeln (Infos unter www.bdh-online.de).

Das Fortbildungszertifikat steht allen Heilpraktikern offen, unabhängig von ihrer Verbandszugehörigkeit.

\section{Homöopathie-Zertifikat:}

\section{Punkte erwerben mit der DHZ}

Die SHZ ist eine gemeinnützige, von Einzelinteressen unabhängige Stiftung des bürgerlichen Rechts.

Sie hat die Aufgabe, ein hohes Qualitätsniveau in der Aus- und Weiterbildung von Homöopathen (Heilpraktikern und Ärzten) zu fördern und zu sichern, mit dem Ziel, Qualitätskriterien für eine klassisch homöopathische Behandlung in der Öffentlichkeit zu etablieren. Die umfassenden Qualifikationsrichtlinien definieren Inhalt und Umfang homöopathischer Ausbildungen (mind. 550 Unterrichtseinheiten Kontaktstudium und 1250 Unter- 


\section{DHZ-Fortbildungspunkte - Fragebogen}

Es ist jeweils nur eine Aussage pro Auswahl korrekt. Bitte wählen Sie bei allen Aussagen die jeweils richtige. Die Aussagen beziehen sich auf die Artikel S. 16-23 in der Rubrik Spektrum in dieser Ausgabe (DHZ 1/2011).

\section{Welche Aussage trifft jeweils zu?}

Auswahl 1

a Das Thoracic-Outlet-Syndrom (TOS) ist ein akutes Kompressionssyndrom.

b Das TOS betrifft die untere Thoraxapertur.

c Das TOS hat ausschließlich neurologische Ursachen.

d Das TOS tritt durch Kompression an anatomischen Engstellen auf.

e Das TOS entsteht durch einen Gefäßverschluss der A. subclavia.

\section{Auswahl 2}

a Das TOS zeigt Beschwerden ausschließlich in Hand und Arm.

b Das TOS zeigt Beschwerden in Hand, Arm und Brust.

c Das TOS zeigt Beschwerden in Hand, Arm und Schulter.

d Das TOS betrifft Solar- und Armplexus.

e Das TOS betrifft mehr Männer als Frauen.

\section{Auswahl 3}

a Die Mm. scaleni entspringen an den Querfortsätzen der Halswirbel.

b Die Mm. scaleni unterstützen vorwiegend die Ausatmung.

c Man unterscheidet M. scalenus anterior, superior und inferior.

d Durch die vordere Scalenuslücke zieht die A. subclavia.

e Der M. sternocleidomastoideus entspringt am Hinterhaupt.

\section{Auswahl 4}

a Das TOS entwickelt sich meist ohne erkennbaren Auslöser.

b Das TOS kann sich perakut zum gefäßchirurgischen Notfall entwickeln.

c Alarmsymptome des TOS-Notfalls sind Rötung, Schwellung und Überwärmung der Extremität.

d Die Diagnostik des TOS ist nicht in der HPPraxis möglich.

e Die Halsrippe ist eine anatomische Variante am 6. Halswirbel.

\section{Auswahl 5}

a Lokale TOS-Symptome sind z. B. Missempfindungen und Lähmungen.

b Neurologische TOS-Symptome sind z. B. Muskelverspannungen an der Halsseite.

c Vegetativ-neurologische TOS-Symptome sind z. B. Schwellungen und Schweregefühl in Hand und Unterarm.

d Arterielle TOS-Symptome sind z. B. Blässe und Kühle bis hin zu punktförmigen Hautnekrosen.

e Venöse TOS-Symptome sind z. B. das Raynaud-Phänomen und vermehrte Schweißbildung.

\section{Auswahl 6}

a Das TOS kann z. B. durch Addison-Tests untersucht werden.

b Wichtige DD des TOS sind z. B. der Pancoast-Tumor, MS sowie Thrombose der V. axillaris oder Verschluss der A. brachialis.

c Ein - selten vorhandener - M. supraclavicularis kann nur röntgenologisch nachgewiesen werden.

d Ein Skalenussyndrom geht immer mit einer Halsrippe einher.

e Ein Kostoklavikularsyndrom gefährdet vor allem den Plexus brachialis.

\section{Auswahl 7}

a Das mittlere Zervikalsyndrom wird zwischen dem 2. und 5. Halswirbel angesiedelt.

b Schwindel tritt beim oberen Zervikalsyndrom nie auf.

c Beim oberen Zervikalsyndrom besteht der Kopfschmerz v. a. im Liegen.

d Beim Zervikalsyndrom ist aufgrund der Anatomie am häufigsten der 1 . Halswirbel betroffen.

e Häufig kompensiert der 2. Halswirbel die bei PC-Arbeit betonte Flexion des Kopfes.

\section{Auswahl 8}

a Beim mittleren Zervikalsyndrom wird meist ein Ziehen im paravertebralen Weichteilgewebe beschrieben.

b Typisch für das mittlere Zervikalsyndrom ist das Gefühl eines überstabilen Halses.

c Wenn Schmerzen in den Bereich der Schulterblätter ausstrahlen, deutet dies auf das untere Zervikalsyndrom hin. d Beschwerden im mittleren HWS-Bereich sollten ausschließlich lokal untersucht und behandelt werden.

e Beschwerden im mittleren HWS-Bereich sollten ausschließlich organbezogen untersucht und behandelt werden.

\section{Auswahl 9}

a Ein Bandscheibenvorfall im unteren Drittel der HWS kann kaum durch bildgebende Verfahren festgestellt werden.

b Patienten bezeichnen meist den oberen HWS-Bereich als „Nacken“.

c Beim unteren Zervikalsyndrom verspürt der Patient punktuelle Schmerzen im 6. und 7. Halswirbelbereich, wenn er den Kopf nach vorn beugt.

d Verantwortlich für Hinterkopfschmerzen sind der N. occipitalis minor oder die Galea apoerotica.

e Beim unteren Zervikalsyndrom kann eine schnelle Rotationsbewegung Schwindel verursachen.

\section{Auswahl 10}

a Langes Sitzen vor dem PC führt immer zu einer Überbelastung des Nackens.

b Der N. phrenicus entspringt im Bereich C 8-Th 1.

c Da der N. phrenicus z. B. Leber und Gallenblase versorgt, sollte bei therapieresistenten Beschwerden an eine Wechselbeziehung zwischen unterer HWS und den vom N. phrenicus versorgten Gebieten gedacht werden.

d Da der N. phrenicus z. B. Uterus und Prostata versorgt, sollte bei therapieresistenten Beschwerden an eine Wechselbeziehung mit der HWS gedacht werden.

e Da der N. phrenicus z. B. Nieren und Blase versorgt, sollte bei therapieresistenten Beschwerden an eine Wechselbeziehung mit der HWS gedacht werden. 


\title{
DHZ-Fortbildungspunkte - Antwortbogen
}

\section{DHZ-Antworten}

Lernerfolgskontrolle. Bitte nur eine Antwort pro Auswahl ankreuzen! Sie können in dieser DHZ-Ausgabe 2 Fortbildungspunkte erzielen.

$\begin{array}{lllllll}\text { Frage } & 1 & \text { a } & \text { b } & \text { c } & \text { d } & \text { C } \\ \text { Frage } & 2 & \text { a } & \text { b } & \text { c } & \text { d } & \text { e } \\ \text { Frage } & 3 & \text { a } & \text { b } & \text { c } & \text { d } & \text { e } \\ \text { Frage } & 4 & \text { a } & \text { b } & \text { c } & \text { d } & \text { e } \\ \text { Frage } & 5 & \text { a } & \text { b } & \text { c } & \text { d } & \text { e }\end{array}$

Frage 6

Frage 7

Frage 8

Frage 9

Frage 10 a b c d 9

a b c c e

a (b) c $\mathrm{e}$

a b c d e

a b c d e

\section{Angaben zur Person}

\author{
Name, Vorname
}

Straße

PLZ | Ort

\section{Feld für DHZ-Wertmarke|Abo-Nummer}

Nicht-Abonnenten bitte hier DHZ-Wertmarke aufkleben. Abonnenten bitte Abonummer eintragen.

\section{Erklärung}

Ich versichere, dass ich die Beantwortung der Fragen selbst und ohne Hilfe durchgeführt habe.
Ort | Datum
Unterschrift

\section{Ihr Ergebnis}

\author{
$\square$ Sie haben \\ Fragen richtig beantwortet.
}

Sie haben

DHZ-Punkte erworben.

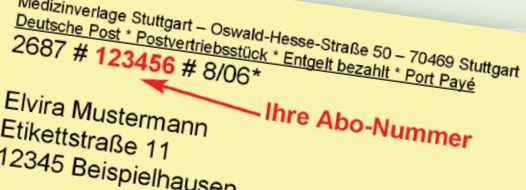

12345 Beise 11

Einsendeschluss 25.02.2012 (Datum des Poststempels)

Bitte senden Sie den vollständigen Antwortbogen zusammen mit einem

an Sie selbst adressierten und ausreichend frankierten Rückumschlag an den 
richtseinheiten Heimstudium). Sie stellen sicher, dass die überprüften Therapeuten die klassische Homöopathie in Theorie und Praxis beherrschen. Für Homöopathen nach der Ausbildung ist neben einer Zertifikatsprüfung eine 3-jährige Supervisionszeit obligatorisch. Mit dem Erhalt des Zertifikats verpflichten sich die zertifizierten Homöopathen, ihr Wissen ständig auf dem Laufenden zu halten. Regelmäßige Fortbildungen auf homöopathischem (30 Unterrichtseinheiten à 45 min pro Jahr) und schulmedizinischem Gebiet (8 Unterrichtseinheiten à 45 min pro Jahr) müssen alle 2 Jahre nachgewiesen werden, um die Zertifizierung aufrechtzuerhalten (Infos unter www.homoeopathie-zertifikat.de).

Die SHZ hat sich seit der Ausgabe DHZ 6/2008 entschieden, allen Zertifikatsinhabern die Chance der interaktiven Fortbildung anzubieten, um den Nachweis für die klinische Fortbildung zu erbringen. So können die Zertifikatsinhaber die Fortbildungspunkte der DHZ bei der SHZ als Nachweis für die klinische Fortbildung einreichen (max. 4 Unterrichtseinheiten pro Jahr - das entspricht 4 Fortbildungspunkten der DHZ).

\section{Verbände und unabhängige Stiftung stehen für Qualität}

Das Entscheidende an diesen beiden Systemen ist, dass Teilnehmer nicht einfach Teilnahmebescheinigungen von Fortbildungen sammeln können, sondern dass eine Zertifizierungsstelle, hier ein Berufsverband bzw. eine Stiftung, für die Qualität der Fortbildung steht und diese überprüft und dokumentiert.

Das DHZ-Team unterstützt den Ansatz der regelmäßigen qualifizierten und dokumentierten Fortbildung. Die interaktive Fortbildung halten wir für ganz besonders geeignet, eine qualifizierte Fortbildung zu belegen, weil hier eine eindeutige Lernerfolgskontrolle besteht. Nur wer die Fragen richtig beantwortet, erhält auch seine Fortbildungspunkte.

\section{So geht's}

Sie finden in jeder DHZ-Ausgabe Fortbildungseinheiten. Diese können Sie - auch unabhängig von der Zertifizierung - regelmäßig nutzen, um Ihren Lernerfolg zu überprüfen und zu dokumentieren. Andererseits können Sie sich diese DHZ-Fortbildungs- punkte auch für die Fortbildungszertifikate der genannten Verbände anerkennen lassen.

\section{Anerkennung der DHZ-Beiträge}

Die Vergabe der Fortbildungspunkte kann direkt durch die DHZ erfolgen.

Pro Lerneinheit erhalten Sie 2 Fortbildungspunkte. Hierfür:

nüssen 7 von 10 Fragen richtig beantwortet sein.

- muss der Antwortbogen vollständig ausgefüllt sein.

- muss im entsprechenden Feld des Antwortbogens eine DHZ-Wertmarke aufgeklebt oder Ihre DHZ-Abo-

Nummer eingetragen sein.

Die Abo-Nummer finden Sie auf dem Adressaufkleber Ihres Heftes.

\section{Beantworten und abschicken!}

$\mathrm{Zu}$ einzelnen Beiträgen der DHZ gibt es in jeder Ausgabe Fragebögen (S. II), die Sie ab 2010 auch im Internet unter www.thiemeconnect.de/ejournals/toc/dhz (auch als Nicht-Abonnent der DHZ) herunterladen und ausdrucken können. Schicken Sie uns einfach den ausgefüllten Antwortbogen (S. III). Wir prüfen Ihre Antworten und benachrichtigen Sie über Ihren Lernerfolg. Die Bescheinigung können Sie dann bei der zentralen Dokumentationsstelle des Fortbildungszertifikats für Heilpraktiker beim $\mathrm{BDH}$ und für das Homöopathiezertifikat bei der SHZ einreichen.

Die Teilnahme ist rückwirkend bis max. 1 Jahr nach Erscheinen der jeweiligen DHZ-Ausgabe möglich. Beachten Sie den Einsendeschluss auf dem Antwortbogen.

\section{Datenschutz}

Ihre Daten werden ausschließlich für die Bearbeitung dieser Fortbildungseinheit verwendet. Es erfolgt beim Verlag keine Speicherung der Ergebnisse über die für die Bearbeitung der Fortbildungseinheit notwendige Zeit hinaus. Die Daten werden nach Versand der Punktbescheinigungen anonymisiert. Namen- und Adressangaben auf den Antwortbögen dienen ausschließlich dem Versand der Punktbescheinigungen. Die Angaben zur Person dienen nur statistischen Zwecken und werden von den Adressangaben getrennt und anonymisiert verarbeitet.

\section{DHZ-Wertmarke für}

\section{Nichtabonnenten}

DHZ-Wertmarken können beim Verlag erworben werden: 6er-Pack DHZ-Wertmarken; Preis 25,00 € inkl. MwSt.; Artikel-Nr. 902999

\section{Bitte richten Sie Ihre Bestellung an: \\ MVS Medizinverlage Stuttgart \\ KundenServiceCenter Buch \\ Postfach 301120; 70451 Stuttgart \\ Tel.: 0711/8931-900}

Dieser Artikel ist online zu finden unter: http://dx.doi.org//10.1055/s-0031-1274911

Teilnahme

Jeder Heilpraktiker soll die Möglichkeit zur Teilnahme an der Fortbildung erhalten. Deshalb ist die Teilnahme an der DHZ-Fortbildung nicht an ein Abonnement geknüpft. Dennoch sollten Sie im Besitz einer $\mathrm{DHZ}$ sein, denn alle im Fragebogen enthaltenen Fragen beziehen sich auf die Spektrumbeiträge der jeweiligen Ausgabe. An diversen Heilpraktikerschulen finden Sie DHZExemplare, die dort ausliegen. Darüber hinaus können Sie als Nicht-Abonnent der DHZ Einzelexemplare erwerben bei: KundenServiceCenter Zeitschriften, Rüdigerstr. 14, 70469 Stuttgart, Tel.: 0711/8931-308. Für Abonnenten ist die Teil-nahme kostenfrei. Nicht-Abonnenten können DHZ-Wertmarken erwerben. 